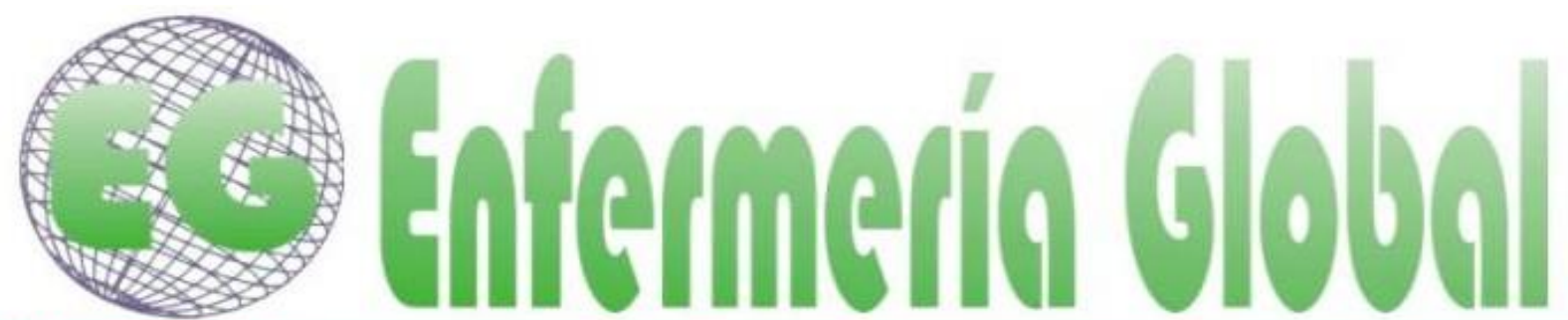

ISSN 1695-6141

$N^{\circ} 47$

Revista electrónica trimestral de Enfermería

Julio 2017

www.um.es/eglobal/

ORIGINALES

\title{
Relación entre el grado de satisfacción y el nivel de estrés identificado en padres y madres con hijos ingresados en una unidad de cuidado intensivo neonatal
}

Relationship between satisfaction and stress levels identified in parents with children admitted to a Neonatal Intensive Care Unit

\author{
$M^{\mathrm{a}}$ José Sánchez-Veracruz ${ }^{1}$ \\ César Leal-Costa ${ }^{2}$ \\ Jesús David Pastor-Rodríguez ${ }^{3}$ \\ José Luis Díaz-Agea ${ }^{4}$
}

\begin{abstract}
${ }^{1}$ Máster en Enfermería de Urgencias, Emergencias y Cuidados Especiales por la Universidad Católica de Murcia (UCAM).

${ }^{1}$ Doctor en Psicología por la Universidad Miguel Hernández. Profesor Facultad de Enfermería. Universidad Católica de Murcia (UCAM).

${ }^{1}$ Máster en Salud, Mujer y cuidados por la Universidad de Murcia. Profesor Facultad de Enfermería. Universidad Católica de Murcia (UCAM).

${ }^{1}$ Doctor en Ciencias Sociosanitarias por la Universidad Católica de Murcia. Profesor Facultad de Enfermería. Universidad Católica de Murcia (UCAM).
\end{abstract}

E-mail: cleal@ucam.edu

http://dx.doi.org/10.6018/eglobal.16.3.256061

Recibido: 09/04/216

Aceptado: 19/07/2016

\section{RESUMEN:}

Objetivos: Identificar la relación existente entre el grado de estrés y el nivel de satisfacción, y analizar qué aspectos influyen en la percepción de estrés general en los padres y madres con hijos ingresados en UCIN.

Método. Estudio descriptivo transversal realizado sobre una muestra de 24 madres/padres. Como instrumentos de evaluación se utilizaron un cuestionario de variables sociobiodemográficas elaborado "ad hoc", la escala de estrés parental: Unidad de Cuidado Intensivo Neonatal (PSS: NICU) y el cuestionario sobre la calidad de la atención hospitalaria (SERVQHOS).

Resultados. Se observan diferencias estadísticamente significativas entre algunas variables sociobiodemográficas y las dimensiones de la escala PSS: NICU y del cuestionario SERVQHOS. Las variables que mejor predijeron el estrés general fueron las dimensiones Aspecto y comportamiento del recién nacido $(A C)$ y papel de la madre $(P M)\left(R^{2}=0,357 ; F(2,21)=5,822 ; p=0,010\right)$.

Conclusiones. Promover una adecuada comunicación entre los profesionales y los padres/madres con hijos ingresados en una UCIN, hace que aumente en ellos el grado de satisfacción; sin embargo, esto no provoca una disminución del estrés general. Las variables que más influyen sobre los niveles de estrés general son las alteraciones en el aspecto/comportamiento del bebé y el papel de la madre.

Palabras clave. Unidad de cuidados intensivos neonatal; estrés; satisfacción; comunicación; padres. 


\section{ABSTRACT:}

Aims. 1) Identify the relationship between the degree of stress and satisfaction, and 2) analyse what aspects influence the overall perception of stress in parents with children admitted to a NICU.

Method. Cross-sectional descriptive study of a sample of 24 mothers / fathers. A socio-biodemographic questionnaire prepared "ad hoc", parental stress scale Neonatal Intensive Care Unit (PSS: NICU) and questionnaire about the quality of hospital care (SERVQHOS) were used.

Results. Statistically significant differences between some socio-biodemographic variables and the dimensions of the PSS:NICU scale and the SERVQHOS questionnaire were observed. The variables that best predict the overall stress were the dimensions appearance and behaviour of the new-born $(A C)$ and the role of the mother $(P M)(R 2=0.357, F(2,21)=5.822 ; p=0.010)$.

Conclusions. Promote proper communication between professionals and fathers / mothers with children admitted to a NICU, increases in them satisfaction; however, this does not cause a decrease in overall stress. The variables that most influence on the overall stress levels are alterations in the appearance / behaviour of the baby and the role of the mother.

Keywords. Neonatal intensive care unit; stress; satisfaction; communication; parents.

\section{INTRODUCCIÓN}

Cuando un neonato ingresa en una Unidad de Cuidados Intensivos Neonatales (UCIN), la mayoría de los esfuerzos, se centran en proporcionar todos los cuidados necesarios para intentar revertir la situación crítica de salud, dejando en un segundo plano las necesidades/sentimientos que pueden experimentar los padres ${ }^{1}$.

Por lo general, se ha identificado a lo largo de la hospitalización, que las madres sufren una urgente necesidad por recuperar su función de cuidadoras, poseen la necesidad de estar con el bebé, de estar bien informadas de su evolución, de cuidarlo, etc. En definitiva, tienen la urgente necesidad de "hacer de madres", recuperando así su rol materno perdido tras el ingreso de su hijo en una $U \mathrm{CIN}^{2-5}$.

El hecho de hacer partícipes a los padres en el cuidado de su hijo hace que vuelvan a recuperar el rol de cuidador principal, produciendo en ellos una sensación de alegría, placer y seguridad, además de disminuir la ansiedad y angustia causada por el ingreso del bebé ${ }^{6,7}$.

Los padres/madres que están pasando por el ingreso de un hijo en una UCIN identifican como necesidades, la información, el contacto con su hijo/a, la identificación con otros padres que estén pasando por la misma situación, la identificación de un profesional de referencia, la comunicación, la intimidad, etc. Cubriendo tan sólo estas necesidades, se podría garantizar que los padres terminarán sintiéndose satisfechos de la atención recibida, ayudándolos así a tener una actitud positiva ante estas situaciones de dificultad ${ }^{5,8}$.

Teniendo en cuenta que los cuidados que se les proporcionan a los bebés han de ser holísticos y que, al tener en cuenta las necesidades de los padres, involucrándolos de forma activa en el proceso, se ayuda, no sólo a la recuperación de los bebés, sino también al bienestar paterno ${ }^{7,9,10}$, se hace necesario conocer el nivel de satisfacción percibido para adecuar estas unidades a las necesidades/sentimientos de los padres, aumentando así la calidad asistencial ${ }^{11}$.

El estrés, según afirma Capdevila et $\mathrm{al}^{8}$, es uno de los parámetros más tenidos en cuenta a la hora de medir y analizar la satisfacción en padres/madres con hijos ingresados en UCIN. Un estudio realizado en Reino Unido ${ }^{12}$, evaluó la escala de estrés parental en una UCIN (PSS: NICU), demostrando la utilidad que posee esta 
escala para medir, no solo el estrés en madres y padres con hijos ingresados en una UCIN, sino también para evaluar la eficacia de la atención de enfermería y servicios de apoyo psicosocial para los padres.

La satisfacción de los pacientes está directamente relacionada con los procesos relacionales asistenciales, donde la labor de enfermería en la humanización de sus cuidados contribuye al bienestar deseado para los padres, representando así una auténtica mejora en la calidad asistencial ${ }^{13}$.

Los objetivos que se plantean en el siguiente estudio son 1) Describir las características sociobiodemográficas de los padres con hijos ingresados en una UCIN y su posible relación con el estrés y la satisfacción percibidas, 2) identificar la relación entre el grado de estrés y el nivel de satisfacción, y 3) analizar qué aspectos predicen mejor el estrés general en los padres.

\section{MÉTODO}

Estudio descriptivo transversal, se llevó a cabo en el Hospital Clínico Universitario "Virgen de la Arrixaca", hospital de referencia en la Región de Murcia, durante los meses marzo-junio del 2015.

\section{Muestra}

Para la realización de éste estudio, se utilizó una muestra total de 24 padres y madres utilizando un muestreo por conveniencia; 8 padres y 16 madres. Los criterios de inclusión fueron 1) todos aquellos padres y madres tuvieran ingresado a un hijo en la UCIN del hospital, y 2) que supieran leer y escribir. Los criterios de exclusión fueron 1) todos aquellos padres y madres que no firmaron el consentimiento informado, y 2) que no rellenaron correctamente los instrumentos de recogida de datos.

\section{Procedimiento}

Una vez obtenido el consentimiento por parte del hospital para la realización del estudio, se procedió a la obtención de la muestra, para ello, durante tres meses se les administró a todas las madres y padres un cuestionario de medida que incluía diferentes instrumentos.

Los sujetos fueron informados detalladamente de los objetivos y condiciones del estudio y se les solicitó el consentimiento informado, tanto para su participación en el mismo, como para la posterior utilización y divulgación de los resultados obtenidos.

Así mismo, se garantizó la total confidencialidad de todos los datos e informaciones relativas a los participantes.

\section{Instrumentos}

Los participantes cumplimentaron un cuestionario autoadministrado que incluía:

- Características sociobiodemográficas de los participantes. Instrumento formado por 17 ítems de respuesta cerrada que, recopila información sobre los aspectos biológicos, sociales y culturales tanto de las madres/ padres como del bebé. Los datos que aparecen en el cuestionario se podrían clasificar en dos grupos: 1) Datos referentes a los padres y madres: edad, la situación laboral, número de hijos, experiencias pasadas en UCIN, el sexo, ingresos económicos mensuales, estado civil, nivel de estudios y nacionalidad. 2) Datos relacionados con el bebé: peso, edad gestacional, tipo de concepción, motivo del ingreso en 
UCIN, días de ingreso, utilización de la ventilación mecánica (VM), tipo de parto y número de abortos previos.

- Escala de estrés parental: Unidad de Cuidado Intensivo Neonatal (PSS: NICU) $)^{3,13-15}$. Esta escala tiene mide la percepción que tienen los padres sobre los factores estresantes derivadas del entorno físico y psicosocial de la unidad de cuidados intensivos neonatales. Fue realizada por Miles, Funk y Carlson ${ }^{14}$, quienes identificaron dentro de la UCIN cuatro factores de estrés ambiental que sirvieron de asiento para la realización de la escala. Las diferentes subescalas de las que consta, miden el nivel de estrés experimentado. Esta escala se basa en la teoría de estrés expuesta por Magnusson ${ }^{13}$, que entiende por estrés la reacción de un individuo a las demandas que ejercen presión sobre sus recursos personales. Consta de 46 ítems que van desde no estresante (1) a extremadamente estresante (5); considerando el 0 como no aplicable (NA), presentando un rango de 46 a 190 puntos. Uno de los problemas que presenta esta escala, viene determinado precisamente por el valor otorgado a la respuesta NA. En la escala, al NA se le otorga el valor "0", con lo que, aun no aumentando la suma total, computa a la hora de introducirlas en un rango, por ejemplo, en el caso de la subescala "imágenes y sonidos" que consta de cinco ítems, si el progenitor responde a cada pregunta con un valor 1 "no ha sido estresante en absoluto", obtendrá una suma igual a cinco, y se incluirá en el grupo de "no estresados". De esta forma, cuando un padre/madre responde con un NA en cuatro de las preguntas y con un 5 en la restante, dicho progenitor pertenecerá al mismo grupo; situación que es totalmente errónea.

- Para evitar esta situación, las respuestas NA, se han tomado como valores perdidos y el resultado total de la suma se ha obtenido a partir del promedio, siendo éste, un modo de resolver las dificultades inherentes a la valoración NA. Las cuatro dimensiones de la escala son: 1) Imágenes y sonidos, definido como entorno físico (incluidas máquinas, equipos, luces...); 2) Apariencia y el comportamiento infantil, que hace referencia a la forma en que los padres aprecian el comportamiento de su hijo; 3) Relación padres-hijo, que se ocupa de la relación padres-bebé y el papel de los padres; y 4) Personal, que se entiende como la comunicación con el personal y la actitud de éstos que aprecian los padres sobre la condición de su bebé o tratamiento que éste recibe. Presenta unas adecuadas propiedades psicométricas, con un coeficiente alpha aceptable, mayor $>0,70$ para todas las subescalas y 0,94 para la escala total.

- Cuestionario de opinión sobre la calidad de la atención hospitalaria $(\text { SERVQHOS })^{16}$. Este instrumento de medida tiene por objetivo evaluar el nivel de calidad percibida por los pacientes. Consta de 19 ítems, de respuesta fácil y rápida, que abarcan los elementos básicos en la evaluación de la calidad percibida y puede ser pasada al paciente, como a los familiares. Los ítems tienen una escala de respuesta tipo likert, que va desde "Mucho peor de lo que esperaba" (1) a "mucho mejor de lo que esperaba" (5) pasando por "peor de lo que esperaba" (2), "como me lo esperaba" (3), "mejor de lo que esperaba" (4). El cuestionario SERVQHOS está construido de tal modo que, a mayor puntuación, mayor nivel de calidad percibida y viceversa; discriminando así, a los pacientes satisfechos de los insatisfechos, basándose en diferentes criterios habitualmente considerados de buena práctica. Se pueden diferenciar en ella dos dimensiones, 1) Calidad Subjetiva: contiene 10 ítems y explica el 
$36,35 \%$ de la varianza (Cronbach de 0,95.), trata aspectos de empatía, capacidad de respuesta, seguridad. 2) Calidad Objetiva: contiene 9 ítems y explica el $28,97 \%$ de la varianza (Alfa de Cronbach 0,89 ), contiene ítems que hacen referencia a situaciones y aspectos susceptibles de comprobación, como limpieza de las habitaciones, estado de conservación, la uniformidad del personal, la puntualidad en las consultas o la información que se facilita al paciente o sus familiares.

\section{Análisis de los datos}

Para procesar la información se elaboró una base de datos con el programa informático SPSS@ v21, realizándose un análisis con diferentes tipos de pruebas. Por un lado se analizaron los estadísticos descriptivos (mínimo, máximo, media, y desviación típica) de las puntuaciones en cada una de las dimensiones de los instrumentos PSS: NICU y SERVQHOS, y las variables sociobiodemográficas cuantitativas, y por otro, frecuencias y porcentajes de las variables cualitativas.

Para estudiar la relación entre las variables estudiadas de interés, se utilizaron las correlaciones bivariadas de Pearson. Para analizar si había diferencias de medias entre las dimensiones de los instrumentos administrados $y$ las variables sociobiodemográficas, se utilizaron la t-student y ANOVA, una vez comprobados los supuestos de normalidad y homogeneidad de las varianzas. También se realizó una regresión lineal múltiple para conocer las variables predictoras de estrés general en los padres.

\section{RESULTADOS}

En la realización de este estudio han participado un total de 8 padres y 16 madres con una edad media de 30,96 años (DT=5,77). En cuanto al estado civil de los progenitores, el $58,4 \%$ declaró estar casado frente al $41,6 \%$ que alegó estar soltero o en situación de convivencia con la pareja. Para el $50 \%$ de los padres, el bebé ingresado en UCIN era su primer hijo, mientras que para la otra mitad, era el segundo. El $12,5 \%$ de los padres declaró tener estudios primarios mientras que el $16,7 \%$ y $20,8 \%$ poseían ciclos formativos superiores y medios respectivamente; el $12,5 \%$ de la muestra declaró no tener estudios, frente al 37,5\% que declaró tener estudios universitarios. El $20,8 \%$ de las parejas sujetas a estudio eran inmigrantes. En cuanto al nivel económico de los padres, destacamos que el $25 \%$ poseían unos ingresos inferiores a $700 €$, frente al $12,5 \%$ que cobraban más de $2000 €$ mensuales. El resto de padres alegó cobrar entre 700-2000€. En relación a la ocupación, el 16,7\% declaró encontrarse en situación de desempleo frente al $62,5 \%$ de padres que se encontraban trabajando, mientras que el 20,8\% estaba formado por amas de casa. Cabe destacar que el $100 \%$ de los padres que participaron en el estudio, declaró haber sido la primera vez que tenían a un hijo ingresado en $U C I N$, habiéndose encontrado una estancia media en UCIN de 23,92 días (DT=28,59).

El $83,3 \%$ de los bebés ingresados requirió, a lo largo del proceso de hospitalización, el uso de ventilación mecánica. En relación al motivo de ingreso, el $62,5 \%$ de los bebés fue ingresado por prematuridad, el $8,3 \%$ por pérdida del bienestar fetal, el $4,2 \%$ por problemas infecciosos y el $25 \%$ por problemas respiratorios. El $12,5 \%$ de los embarazos se realizó a través de fecundación asistida frente al $87,5 \%$ que fue concebido de forma natural. Respecto a la edad gestacional, 10 niños nacieron entre la 26 y 30 semanas de gestación $(41,7 \%) ; 4$ nacieron entre la semana 36 y 37 $(16,7 \%)$, y 10 entre 31 y 35 semanas de gestación (41,7\%). En cuanto al peso al 
nacer, 9 niños $(37,5 \%)$ pesaron menos de 2500 gramos, $6(25 \%)$ pesaron menos de 1500 gramos, 8 (33,3\%) pesaron al nacer menos de 1000 gramos y, tan sólo 1 niño $(4,2 \%)$ tuvo un peso menor de 500 gramos. El 91,7\% de la muestra no había sufrido abortos previos, mientras que el 8,3\% declaró haber tenido, previo al nacimiento del bebé, algún aborto. En cuanto al tipo de parto, el 62,5\% de los padres tuvieron un parto eutócico, frente al $37,5 \%$ que tuvieron que ser intervenidos en el momento del parto necesitando una cesárea urgente.

Los resultados arrojados en las dimensiones de la Escala Estrés Parental (PSS: NICU), los padres y madres obtuvieron una puntuación media-baja en las dimensiones vistas y sonidos (V/S), y comportamiento y comunicación del personal $(\mathrm{C} / \mathrm{C})$; y una puntuación media-alta, en las dimensiones aspecto y comportamiento de su bebé $(A / C)$, y relación con su bebé y su papel de madre/padre $(P / M)$. En cuanto al ítem final de la escala que mide el estrés general experimentado por los padres/madres durante el periodo de hospitalización de sus hijos en la UCIN, obtuvieron una puntuación alta (Tabla 1).

En relación a las puntuaciones obtenidas en las dimensiones que forman la encuesta SERVQHOS, encontramos que los 24 padres/madres obtuvieron una puntuación media-alta en las dos dimensiones del cuestionario (Tabla 1).

Tabla 1 Estadísticos descriptivos (mínimo, máximo, media y desviación típica) de las dimensiones de la escala de estrés parental.

\begin{tabular}{lccccc}
\hline & N & Mínimo & Máximo & M & DT \\
\hline V/S & 24 & 1 & 4 & 2,25 & 0,60 \\
A/C & 24 & 2 & 4 & 2,87 & 0,79 \\
P/M & 24 & 1,22 & 4,88 & 3,14 & 1,14 \\
C/C & 19 & 1,00 & 4,30 & 2,27 & 0,98 \\
E/G & 24 & 1 & 5 & 3,46 & 1,44 \\
C_OBJ & 24 & 2,78 & 5,00 & 4,11 & 0,64 \\
C_SUB & 24 & 2,80 & 5,00 & 4,31 & 0,67 \\
\hline
\end{tabular}

N: número de participantes; M: Media; DT: Desviación Típica; V/S: dimensión vistas y sonidos; A/C: dimensión aspecto y comportamiento; $\mathrm{P} / \mathrm{M}$ : dimensión papel de la madre; C/C: dimensión comportamiento y comunicación del personal; E/G: estrés general; C_OBJ: dimensión calidad objetiva; C_SUB: dimensión calidad subjetiva.

Los resultados obtenidos en el análisis de las correlaciones bivariantes, arrojaron una correlación negativa y estadísticamente significativa entre las dimensiones calidad subjetiva y calidad objetiva y la dimensión comportamiento y comunicación del personal $(\mathrm{C} / \mathrm{C})$. Por otro lado, también se observa, una correlación positiva y estadísticamente significativa entre el estrés general $(E / G)$ y la dimensión vistas y sonidos (V/S), aspecto y comportamiento del bebe $(\mathrm{A} / \mathrm{C})$, papel de la madre $(\mathrm{P} / \mathrm{M})$, y calidad objetiva (C_OBJ) (Tabla 2). 
Tabla 2. Correlaciones bivariadas de Pearson entre las variables edad, días de hospitalización, las dimensiones y el último ítem de la escala de estrés parental y las dimensiones de la encuesta SERVQHOS.

\begin{tabular}{|c|c|c|c|c|c|c|c|c|c|}
\hline & Edad & D hosp & $\mathrm{V} / \mathrm{S}$ & $\mathrm{A} / \mathrm{C}$ & $\mathrm{P} / \mathrm{M}$ & $\mathrm{C} / \mathrm{C}$ & $E / G$ & $\mathrm{C}$ OBJ & C_SUB \\
\hline $\begin{array}{l}\text { Edad } \\
\text { D }\end{array}$ & .070 & -- & & & & & & & \\
\hline V/S &,- 019 & 013 & -- & & & & & & \\
\hline $\mathrm{A} / \mathrm{C}$ & 017 & 080 &,- 061 & -- & & & & & \\
\hline $\mathrm{P} / \mathrm{M}$ & ,242 &,- 027 &, 131 & , 424 & -- & & & & \\
\hline $\mathrm{C} / \mathrm{C}$ &,$- 363^{*}$ &,- 089 &,- 160 & 024 & , 166 & -- & & & \\
\hline$E / G$ & ,227 &, 150 &, $363^{*}$ &, $344^{*}$ &, $588^{\mathrm{x}}$ & ,116 & 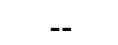 & & \\
\hline C_OB & 147 &,- 131 &,- 060 & ,025 &,- 140 &,$- 393^{* x}$ &,$- 396^{* \pi}$ & -- & \\
\hline C SU & $249^{*}$ & -094 & 127 & -.073 &,- 108 &,$- 490^{* x}$ & 049 & ,670 & -- \\
\hline
\end{tabular}

* $\mathrm{p}<0,05, " \mathrm{p}<0,01$.

D hosp: Días de hospitalización; V/S: dimensión vistas y sonidos; A/C: dimensión aspecto y comportamiento; P/M: dimensión papel de la madre; C/C: dimensión comportamiento y comunicación del personal; E/G: estrés general; C_OBJ: dimensión calidad objetiva; C_SUB: dimensión calidad subjetiva.

En cuanto a los resultados obtenidos mediante la prueba t-student entre las dimensiones de los instrumentos administrados y las variables sociobiodemográficas categóricas de dos niveles, se encontraron diferencias estadísticamente significativas entre la variable nacionalidad y las dimensiones $P / M, E / G$, calidad objetiva y calidad subjetiva; obteniendo una $\mathrm{t}(22)=2,741 ; \mathrm{p}=0,012$ para la dimensión $\mathrm{P} / \mathrm{M}$, una $\mathrm{t}(22)=$ 3,$534 ; p=0,002 ; \mathrm{IC}(0,866 ; 3,324)$ para la dimensión $E / G$, una $t(22)=-2,508 ; p=0,020$; IC $(-1,33327 ;-0,12638)$ para calidad objetiva y una $t(22)=-2,161 ; p=0,042$; IC (1,31618; -0,02698) para calidad subjetiva. Así los padres de nacionalidad no española tuvieron puntuaciones más altas en las dimensiones calidad objetiva/subjetiva, y presentaron menores niveles de estrés general $(E / G)$ y en la dimensión papel de la madre $(P / M)$

En la variable ventilación mecánica, se observaron diferencias estadísticamente significativas con la dimensión calidad subjetiva, con una $t(22)=2,608 ; p=0,016$; IC $(0,17407 ; 1,52593)$, teniendo mayor calidad subjetiva los padres cuyos hijos precisaron asistencia con VM.

Por último, la dimensión estrés en general $(E / G)$, también presentó diferencias estadísticamente significativas con la variable tipo de concepción con una $\mathrm{t}(22)=-$ 2,$122 ; p=0,045 ;$ IC $(-3,484 ;-0,040)$, presentando más estrés los padres que habían concebido a sus hijos mediante fecundación asistida.

En cuanto a los resultados obtenidos mediante la prueba estadística ANOVA realizada, entre las dimensiones de los instrumentos administrados y las variables sociobiodemográficas categóricas de más de dos niveles, se encontraron diferencias estadísticamente significativas entre la variable nivel económico y la dimensión $\mathrm{C} / \mathrm{C}$ $F(4)=4,110 ; p=0,021$. Las comparaciones múltiples, realizadas en el análisis post hoc, indicaron que sólo existieron diferencias estadísticamente significativas entre los padres que cobran $<700 €$ y los que cobraban $1500-2000 €$.

También se encontraron diferencias estadísticamente significativas entre la variable ocupación y la dimensión $\mathrm{C} / \mathrm{C}$, presentando una $\mathrm{F}(2)=4,063 ; \mathrm{p}=0,037$. Las comparaciones múltiples, realizadas en el análisis post hoc, señalaron diferencias 
estadísticamente significativas entre los padres y madres que conservaban su trabajo y los que estaban en situación de desempleo.

En cuanto a la variable semanas de gestación, se encontraron diferencias estadísticamente significativas con la dimensión $\mathrm{P} / \mathrm{M}$ de la escala de estrés, con una $F(2)=3,989 ; p=0,034$. Tras la realización de las comparaciones múltiples, comprobamos que los padres/madres con hijos nacidos entre la semana 36-37 tienen más estrés que los nacidos en la semana 31-35.

Para finalizar, se efectuó un análisis de regresión lineal múltiple, en los que se utilizaron las dimensiones de la escala de estrés parental (V/S, A/C, P/M y C/C) como variables predictoras, y la variable estrés general (ítem estrés general durante la estancia en la UCIN) como variable dependiente. Así, las variables que mejor predijeron el estrés general fueron las dimensiones Aspecto y comportamiento del recién nacido $(A C)$ y papel de la madre $(P M) \quad\left(R^{2}=0,357 ; F(2,21)=5,822 ; p=0,010\right)$ (Tabla 3).

Tabla 3. Análisis de regresión lineal múltiple sobre ítem estrés general durante la estancia en la UCIN.

\begin{tabular}{lccccc}
\hline & $\mathbf{R}^{\mathbf{2}}(\%)$ & $\mathbf{F}$ & $\mathbf{B}$ & $\mathbf{t}$ & $\mathbf{p}$ \\
\hline VD: estrés general & 35,7 & 5,822 & & & 0,010 \\
Constante & & & 1,708 & 2,056 & 0,009 \\
A/C & & & 0,307 & 1,410 & 0,046 \\
P/M & & & 0,681 & 2,789 & 0,003 \\
\hline
\end{tabular}

A/C: dimensión aspecto y comportamiento; P/M: dimensión papel de la madre

\section{DISCUSIÓN}

En este estudio se plantea analizar la relación existente entre el grado de estrés y el nivel de satisfacción experimentado por padres y madres con hijos ingresados en una UCIN, además de examinar la relación de las variables sociobiodemográficas de los progenitores sobre los niveles de estrés y satisfacción.

En cuanto a los datos obtenidos sobre la escala de estrés parental y su relación con las variables sociobiodemograficas, destacamos que en función de las variables, nacionalidad, ocupación, tipo de concepción, nivel económico y semanas de gestación del bebé, los niveles de estrés varían. Sin embargo, las variables edad del progenitor, estado civil, nivel cultural, número de hijos, abortos previos, tipo de parto, peso, ventilación mecánica, días de ingreso y motivo de ingreso no presentaron diferencias estadísticamente significativas sobre el estrés. En ello coinciden autores como Mackley et $\mathrm{al}^{17}$, quienes afirmaron que el matrimonio o el nivel cultural no tiene influencia sobre los niveles de estrés identificados en las madres/padres con hijos ingresados en UCIN. En contraposición, Alkozei et al $^{18}$ encontraron, sobre una muestra de 85 sujetos, diferencias estadísticamente significativas en el estado civil, puntuando más alto los padres casados en las dimensiones papel de la madre $(P / M)$ y vistas y sonidos (V/S). Recientemente, y a diferencia de nuestro estudio, se ha asociado la variable edad avanzada de los progenitores o nacimiento muy prematuro del bebé, a mayores niveles de estrés ${ }^{19}$. En cuanto a la variable sexo, no se han encontrado diferencias significativas entre madres y padres, coincidiendo así con otros autores como Aftyka et $\mathrm{al}^{20}$. Por otro lado, en el trabajo realizado por Chourasia et $\mathrm{al}^{21}$, se encontraron diferencias estadísticamente significativas en los niveles de 
estrés en función de la edad materna, la prematuridad del bebé y una estancia previa en UCIN, coincidiendo así con nuestro estudio, en la prematuridad del bebé. En cuanto a la variable, estancia previa en UCIN, nuestros datos no son equiparables al citado estudio puesto que el $100 \%$ de nuestra muestra obtenida alegó no haber tenido un ingreso previo en UCIN.

En lo referente a variables sociodemográficas y nivel de satisfacción, se observaron diferencias estadísticamente significativas entre ambas dimensiones de la encuesta SERVQHOS y las variables nacionalidad presentando mayores niveles de calidad subjetiva y objetiva los padres no españoles. Los padres de los niños que necesitaron el uso de ventilación mecánica percibieron también, mejores niveles de calidad subjetiva, no interfiriendo en la calidad percibida por los padres, por ejemplo, la gravedad de los prematuros, resultados que coinciden con el estudio de Capdevila et $\mathrm{al}^{8}$.

En cuanto a los datos obtenidos sobre los niveles de estrés identificados en las dimensiones que forman la escala PSS: NICU, destacamos que las dimensiones que mejor predijeron los niveles de estrés fueron papel de la madre $(P / M)$ y aspecto y comportamiento del bebé $(\mathrm{A} / \mathrm{C})$ (Tabla 3$)$. En ello, coinciden numerosos autores como, Chourasia et $\mathrm{al}^{22}$, Sikorova et $\mathrm{al}^{23}$ y Boute at $\mathrm{al}^{24}$, quienes también sitúan como principales fuentes de estrés las alteraciones producidas en el rol materno-filial y dentro de ésta, la separación física ocasionada entre madre e hijo.

Por otro lado, González-Escobar et $\mathrm{al}^{4}$ realizaron un estudio sobre una muestra total de 145 madres, no encontrando una relación estadísticamente significativa entre la dimensión comunicación con el personal y el estrés total; según dichos autores a las madres poco les importa el vínculo o relación que puedan mantener con el equipo de salud debido, en gran parte, al poco tiempo que éstas poseen para compartir con sus hijos, enfocándose prioritariamente en estar con estos. Estos resultados son congruentes con nuestro trabajo, ya que no se correlaciona la dimensión comunicación y comportamiento $(\mathrm{C} / \mathrm{C})$ y calidad subjetiva con el ítem estrés general. Los ítems que forman la dimensión $\mathrm{C} / \mathrm{C}$ de los profesionales sanitarios en la escala estrés parental están redactados en negativo, así cuanto mejor es el trato por parte de los profesionales sanitarios, menor es el estrés percibido por los padres en esta dimensión, sin embargo, en la dimensión calidad subjetiva de la SERVQHOS es al revés. Por ello, se encontraron correlaciones estadísticamente significativas y negativas entre la dimensión $\mathrm{C} / \mathrm{C}$ del personal y calidad subjetiva (Tabla 2).

La dimensión calidad subjetiva del cuestionario SERVQHOS, abarca todas las cuestiones relacionadas con la comunicación y el trato por parte de los profesionales; lo que nos indica que, aunque los padres tengan un nivel de estrés elevado debido al ingreso de sus hijos en una UCIN, el nivel de satisfacción es elevado debido al trato recibido, a las habilidades de comunicación del personal sanitario, etc, siendo la calidad asistencial percibida elevada (Tabla 1). Autores como Wigert, et al ${ }^{25}$ encontraron que la comunicación entre los padres y el personal de la UCIN es una parte esencial y puede reducir su estrés emocional, coincidiendo así con los datos obtenidos en nuestro estudio, ya que se ha observado una puntuación baja en la dimensión $\mathrm{C} / \mathrm{C}$ y elevada en calidad subjetiva.

De este modo, aunque parece que no existe una relación entre los aspectos relacionales del personal sanitario de las UCIN con el estrés general que padecen los padres que tienen a sus hijos ingresados, parece que un adecuado trato, mostrar 
empatía, respeto, etc, amortigua el estrés emocional que sufren los padres, y por tanto, aumenta la satisfacción y la calidad asistencial percibida.

\section{CONCLUSIONES}

Las principales conclusiones que se extraen del presente trabajo son:

- Aspectos como el nivel económico, la nacionalidad, la ocupación, el tipo de concepción y la prematuridad influyen sobre los niveles de estrés percibidos por los padres. Por otro lado, la nacionalidad, el número de hijos y el uso de ventilación mecánica durante la estancia en UCIN, se asocian con mayores niveles de satisfacción.

- Promover una adecuada comunicación entre los profesionales y los padres/madres con hijos ingresados en una UCIN, hace que aumente en ellos, el grado de satisfacción; sin embargo, esto no provoca una disminución del estrés general percibido.

- Las variables que más influyen sobre los niveles de estrés general son las alteraciones en el aspecto/comportamiento del bebé y el papel de la madre.

\section{REFERENCIAS}

1. García-Puga JA, Quintana-Zavala MO, Yesenia Acuña-Ruiz MJ, Montoya-Barnez MC. Estrés en Padres con Hijos Hospitalizados en las Unidades de Cuidados Intensivos Pediátricos y Neonatales. Bol Clin Hosp Infant Edo Son. 2009; 26(2): 6772.

2. Cañas Lopera EM. Significado que le asigna el padre a la hospitalización de su hijo recién nacido pretérmino extremo en la unidad de cuidado intensivo neonatal. Bogotá: Universidad Nacional de Colombia; 2012.

3. Cano Giménez E. Estrés y Ansiedad Parental en la Unidad de Cuidados Intensivos Neonatal. El Papel Modulador de una Intervención Temprana [Tesis doctoral]. Universidad de Murcia; 2013.

4. González Escobar DS, Ballesteros Celis NE, Serrano Reatiga MF. Determinants stressors in mothers of preterm infants hospitalized in Intensive Care Units. Rev Cien Cuid. 2012; 9.

5. Cano Moroba S, Marsellés Vidal M A, Jové Monclús G. Un paseo por la 5a planta. Análisis de las vivencias y el grado de satisfacción de los padres de bebés hospitalizados en la UCIN del hospital universitario Arnau de Villanueva de Lleida. Rev Educ Inclus. 2012; 5 (3): 17-32.

6. Martins Faria Faddul Alves MV, Gonzaga Cordeiro J, Bronzato Luppi CH, Trevizani Nitsche MJ y Leite Rosa Olbrich SR. Experience of family members as a result of children's hospitalization at the Intensive Care Unit. Invest Educ Enferm. 2013: 31(2).

7. Achury DM, Achury Beltrán LF, Ramírez Cárdenas AM. La familia y las visitas en las unidades de cuidado intensivo. World Crit Care Nur. 2011; 8(1): 2-9.

8. Capdevila-Cogula E, Sánchez-Pozón L, Riba-García M, Moriña-Soler D, RíosGuillermo J, Porta-Ribera R et al. Valoración de la satisfacción de los padres en una unidad neonatal. An Pediatr (Barc). 2012;77(1):12-21.

9. Martins-Faria F, Alves MV, Gonzaga-Cordeiro J, Bronzato-Luppi CH, TrevizaniNitsche MJ, Leite-Rosa SR. Experience of family members as a result of children's hospitalization at the Intensive Care Unit. Invest Educ Enferm. 2013: 31(2). 
10. Amaral-Martins LA, Santana-da Silva D, De Souza-Azevedo AC, Cedraz-Morais A. Insertion of the family in the neonatal intensive care unit: a systematic review. Rev enferm UFPE. 2012; 6(4): 876-83.

11. Moreno Reyes $P$. Perceptions from parents about the quality of clinical information received from health professionals caring their children in a level III hospital's neonatal ICU, in Cali, Colombia (February - march, 2010). Ciencia \& Salud. 2013; 2(6): 43-49.

12. Franck LS, Cox S, Allen A, Winter I. Measuring neonatal intensive care unit-related parental stress. J Adv Nurs 2005 Mar; 49 (6): 608-15.

13. Magnusson, D. Situational determinants of stress: an interactional perspective. New York: Goldberg Breznitz; 1982.

14. Miles MS, Funk SG, Carlson J. Parental stressor scale: neonatal intensive care unit. Nur Res. 1993; 42: 148-152.

15. Molina RCM, Fonseca EL, Waidman MAP, Marcon SS. La percepción de la familia de su presencia en una Unidad de Cuidados Intensivos Pediátricos y Neonatales. Rev Esc Enferm USP. 2009; 43 (3): 630-8.

16. Mira JJ, Aranaz J, Rodríguez-Marín J, Buil JA, Castell M, Vitaller J. SERVQHOS: un cuestionario para evaluar la calidad percibida de la asistencia hospitalaria. Med Prev. 1998; 4: 12-8.

17. Mackley AB, Locke RG, Spear ML, Joseph R. Forgotten parent: NICU paternal emotional response. Adv Neonatal Care. 2010 Aug; 10(4): 200-3.

18. Alkozei A, McMahon E, Lahav A. Stress levels and depressive symptoms in NICU mothers in the early postpartum period. J Matern Fetal Neonatal Med. 2014 Nov; 27(17): 1738-43.

19. Turner M, Chur-Hansen A, Winefield $H$, Stanners $M$. The assessment of parental stress and support in the neonatal intensive care unit using the Parent Stress Scale Neonatal Intensive Care Unit. Women Birth. 2015; 28(3): 252-8.

20. Aftyka A, Rybojad B, Rozalska-Walaszek I, Rzonca P, Humeniuk E. Trastorno de estrés Post-traumático en los padres de niños hospitalizados en Unidades de Cuidados Intensivos (UCIN): factores de riesgo médico y demográfico. Psychiatr Danub. 2014; 26 (4): 347-52.

21. Chourasia N, Surianarayanan P, Adhisivam B, Vishnu Bhat B. NICU admissions and maternal stress levels. Indian J Pediatr. 2013; 80(5): 380-4.

22. Chourasia N, Surianarayanan P, Bethou A, Bhat V. Stressors of NICU mothers and the effect of counseling-experience from a tertiary care teaching hospital, India. J Matern Fetal Neonatal Med. 2013; 26(6): 616-8.

23. Sikorova L, Kucova J. The needs of mothers to newborns hospitalized in intensive care units. Biomed Pap Med Fac Univ Palacky Olomouc Czech Repub. 2012; 156 (4): 330-6.

24. Boute KM, Claudio N, Ramirez V, García-Fragoso L. La pérdida de la función de los pres como una causa de estrés en la unidad de cuidados intensivos neonatales. Bol Asoc Med PR. 2012; 104 (1): 8-11.

25. Wigert H, Dellenmark Blom M, Bry K. Parents' experiences of communication with neonatal intensive-care unit staff: an interview study. BMC Pediatr. 2014; 14(1): 304.

\section{ISSN 1695-6141}

@ COPYRIGHT Servicio de Publicaciones - Universidad de Murcia 